\title{
AN ANALYSIS OF CARRIER-MEDIATED PHOTODIFFUSION MEMBRANES
}

\author{
RAVI JAIN and JEROME S. SCHULTZ*
}

Department of Chemical Engineering, The University of Michigan, Ann Arbor, MI 48109 (U.S.A.)

(Received January 11, 1983; accepted in revised form February 23, 1983)

\section{Summary}

Reversible photochromic reactions can be combined with carrier-mediated transport in partially illuminated membranes to achieve very high degrees of flux enhancement. Transport across these membranes can be achieved even against a concentration gradient. Coupled systems of non-linear diffusion-reaction equations for these membranes were solved using the technique of orthogonal collocation on finite elements to study and optimize the transport across them. The results indicate that the efficiency of light utilization is maximum for thin membranes at low degrees of illumination. Carrier-mediated photodiffusion membranes in combination with a fuel cell can be used for the storage of solar energy.

\section{Introduction}

Carrier-mediated photodiffusion membranes combine reversible photochromic reactions with carrier-mediated transport in the membrane. The idea of carrier-mediated photodiffusion membranes was proposed by Schultz [1]. In these membranes, a reversible photochromic reaction between a permeant and a carrier takes place in a membrane with different levels of illumination in regions parallel to the surface of the membrane. This arrangement can lead to increased transport of the permeant over that obtained if the membrane is uniformly illuminated or totally dark. In this paper, a numerical analysis of the transport in carrier-mediated photodiffusion membranes is presented. The model system selected for the purposes of illustrating the effects of various parameters is the transport of carbon monoxide across membranes containing hemoprotein solution.

Various parameters studied were the upstream and downstream permeant concentrations, membrane thickness, fraction of membrane illuminated and the ratio of the apparent equilibrium constant for the reaction in light to equilibrium constant in dark. The method of orthogonal collocation on finite elements, reported previously by Jain and Schultz [2], was used to solve the

\footnotetext{
*To whom correspondence should be addressed.
} 
diffusion-reaction equations for this system. The results of these calculations are used to indicate how the transport process in photodiffusion membranes can be optimized. The techniques developed should be applicable to transport in other composite membranes such as those discussed by Donaldson and Quinn [3] and Schultz [4] . Carrier-mediated photodiffusion membranes can also be used for storage of solar energy because in these membranes the transport can be achieved even against a concentration gradient, and in the limiting case of zero flux, a concentration gradient of the permeant can be supported by the action of light energy alone. This concentration gradient can be used to run a fuel cell, thereby storing the solar energy.

\section{Theory}

A schematic diagram of the carrier-mediated photodiffusion membrane is shown in Fig. 1. The region $L_{\mathrm{i}} \leqslant x \leqslant L$ is illuminated, the other portion of the membrane is in the dark. This arrangement can be experimentally achieved by constructing a membrane sandwich consisting of two transparent membranes separated by an opaque barrier to block the light from entering the

DARK LIGHT

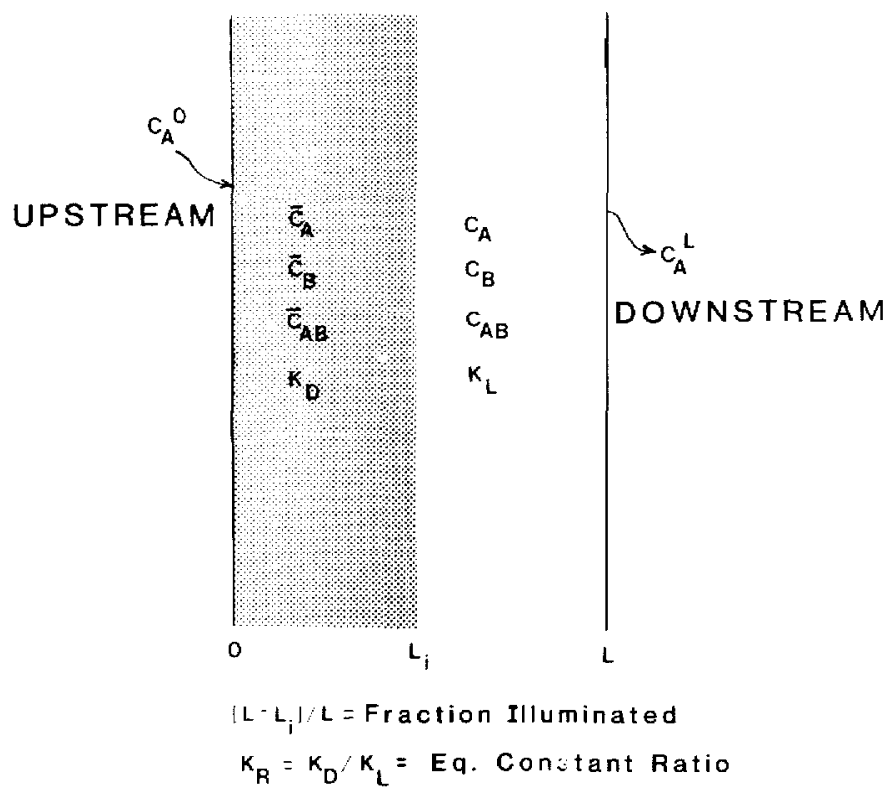

Fig. 1. Schematic diagram of a photodiffusion membrane showing dark ( 0 to $L_{\mathbf{i}}$ ) and illuminated ( $L_{\mathrm{i}}$ to $L$ ) regions. 
dark side of the membrane. A reversible chemical reaction of the form shown below takes place in both regions of the membrane

$$
\mathrm{A}+\mathrm{B} \underset{k_{2}(I)}{\stackrel{k_{1}}{\rightleftharpoons}} \mathrm{AB}
$$

where $A$ is the permeant and $B$ is a carrier which is constrained to remain within the membrane. The reverse rate constant of the reaction, $k_{2}(I)$, is dependent on the light intensity, $I$. For the myoglobin-carbon monoxide system,

$k_{2}(I)=k_{2}(0)+\lambda I$

where $k_{2}(0)$ is the reverse rate constant in dark, $I$ is the light intensity and $\lambda$ is a proportionality constant [5]. The effect of light, then, is to increase the rate constant for the reverse reaction, and hence to decrease the apparent equilibrium constant, $K_{\mathrm{L}}=k_{1} / k_{2}(I)$, for the system. The apparent equilibrium constant for $\mathrm{CO}-\mathrm{Mb}$ system is given by $K_{\mathrm{L}}=k_{1} /\left(k_{2}(0)+\lambda I\right)$. The apparent equilibrium constant refers to the ratio $\left(C_{\mathrm{AB}} / C_{\mathrm{A}} C_{\mathrm{B}}\right)$ in the "photostationary" state. This ratio decreases because of the formation of an active intermediate, which subsequently reacts in the presence of light. The concentrations of the various species in the photostationary state are dependent on light intensity. This effect disappears when the system is brought to the dark state and the concentrations relax to the true thermodynamic equilibrium relationship, $K_{\mathrm{D}}=k_{1} / k_{2}(0)=\left(C_{\mathrm{AB}} / C_{\mathrm{A}} C_{\mathrm{B}}\right)$.

The diffusion-reaction equations describing the system are written below. In the dark region from $x=0$ to $L_{i}$, indicated by overbars,

$D_{i} \frac{\mathrm{d}^{2} \bar{C}_{i}}{\mathrm{~d} x^{2}}=\bar{r}_{i}, \quad i=\mathrm{A}, \mathrm{B}, \mathrm{AB}$

where

$\bar{r}_{\mathrm{A}}=\bar{r}_{\mathrm{B}}=-\bar{r}_{\mathrm{AB}}=k_{1} \bar{C}_{\mathrm{A}} \bar{C}_{\mathrm{B}}-k_{2} \bar{C}_{\mathrm{AB}}$

and in the illuminated region from $x=L_{\mathrm{i}}$ to $L$,

$D_{i} \frac{\mathrm{d}^{2} C_{i}}{\mathrm{~d} x^{2}}=r_{i}, \quad i=\mathrm{A}, \mathrm{B}, \mathrm{AB}$

with

$r_{\mathrm{A}}=r_{\mathrm{B}}=-r_{\mathrm{AB}}=k_{1} C_{\mathrm{A}} C_{\mathrm{B}}-k_{2}(I) C_{\mathrm{AB}}$.

The symbols $D_{i}, r_{i}$ and $C_{i}$ in the equations above refer to diffusivities, reaction rates, and concentrations, respectively. Equation (4) assumes that the illumination is uniform on the illuminated side of the membrane, and hence $k_{2}$ is constant in this region. This would be the case if the amount of light absorbed by the solution is small compared to the total amount of incident illumination. 
The boundary concentrations of the permeant, $\mathrm{A}$, are presumed to be known and components $\mathrm{B}$ and $\mathrm{AB}$ are constrained to stay within the membrane, so we have:

$\begin{array}{ll}C_{\mathrm{A}}=C_{\mathrm{A}}^{o} & \text { at } x=0 \\ C_{\mathrm{A}}=C_{\mathrm{A}}^{L} & \text { at } x=L \\ \frac{\mathrm{d} C_{\mathrm{B}}}{\mathrm{d} x}=0 & \text { at } x=0 \\ \frac{\mathrm{d} \bar{C}_{\mathrm{B}}}{\mathrm{d} x}=0 & \text { at } x=L\end{array}$

where $C_{\mathrm{A}}^{0}$ and $C_{\mathrm{A}}^{L}$ are the concentrations of $\mathrm{A}$ at $x=0$ and $x=L$, respectively. The concentrations and the fluxes have to be continuous across the interface, giving additional boundary conditions,

$C_{\mathrm{A}}=\bar{C}_{\mathrm{A}}, C_{\mathrm{B}}=\bar{C}_{\mathrm{B}}, \frac{\mathrm{d} C_{\mathrm{B}}}{\mathrm{d} x}=\frac{\mathrm{d} \bar{C}_{\mathrm{B}}}{\mathrm{d} x}$, and $\frac{\mathrm{d} C_{\mathrm{A}}}{\mathrm{d} x}=\frac{\mathrm{d} \bar{C}_{\mathrm{A}}}{\mathrm{d} x}$ at $x=L_{\mathrm{i}}$.

Equations (1)-(4), with the boundary conditions given by eqns. (5) and (6), need be solved only for the components $\mathrm{A}$ and $\mathrm{B}$ if the diffusivities of the carrier, $D_{\mathrm{B}}$, and its complex, $D_{\mathrm{AB}}$, are equal. Although for the numerical solution of these equations this assumption is really not needed, as shown by Jain and Schultz [2], for the myoglobin-carbon monoxide system this assumption incurs very little error and we will assume it to hold here for convenience. With this assumption, an algebraic relation is obtained between $C_{\mathrm{B}}, C_{\mathrm{AB}}$ and $C_{\mathrm{T}}$, the total carrier concentration in all its forms. This relation is given by eqn. (7) below [6]:

$C_{\mathrm{B}}+C_{\mathrm{AB}}=C_{\mathrm{T}}, \quad$ for all $x$.

The equations discussed above were converted to dimensionless form before solving. The concentrations were made dimensionless with respect to the permeant concentration at $x=0, C_{\mathrm{A}}^{0}$, and the distances with respect to $L$. The technique was discussed in detail in a previous paper [2] .

\section{Method of solution}

In general, eqns. (1)-(4) and (7) with the boundary conditions given by eqns. (5) and (6) represent a system of second order non-linear differential equations of boundary value type for which no analytical solutions exist. Some physical insight about transport in carrier-mediated membranes can be gained by a dimensionless parameter called the Damköhler number, which is defined by the relation, $N_{\mathrm{D}}=k_{2} L^{2} / D_{\mathrm{A}}$. This parameter is obtained when the transport equations are made dimensionless. If $k_{2}$ and $D_{\mathrm{A}}$ are fixed, the Damköhler number can be changed by changing the membrane thickness, $L$. 
For low values of Damköhler number (thin membranes), the transport is "diffusion" controlled. For large values of Damköhler numbcr (thick membranes), the transport is "reaction" controlled. In the limiting case of a very large Damköhler number, the system is in chemical equilibrium and the net chemical reaction is zero every where in the membrane. This regime of transport is referred to as the "equilibrium" regime. For the photodiffusion membranes this corresponds to the case in which the illuminated part of the membrane is in photostationary state and the dark part is in chemical equilibrium. The facilitation is very small for the "diffusion" regime while it is maximum for the "equilibrium" regime [7] . A closed form solution can be obtained for the "equilibrium" regime and is given by eqn. (8) below [1].

$N_{\mathrm{A}}=\frac{D_{\mathrm{A}}\left(C_{\mathrm{A}}^{0}-C_{\mathrm{A}}^{L}\right)}{L}+\frac{D_{\mathrm{AB}} C_{\mathrm{T}}}{L}\left[\frac{C_{\mathrm{A}}^{o}}{K_{\mathrm{D}}^{-1}+C_{\mathrm{A}}^{0}}-\frac{C_{\mathrm{A}}^{L}}{K_{\mathrm{L}}^{-1}+C_{\mathrm{A}}^{L}}\right]$

where $K_{\mathrm{L}}$ and $K_{\mathrm{D}}$ are the apparent equilibrium constants in the light and true equilibrium constant in dark side of the membrane. Of course, the equilibrium constant, $K_{\mathrm{L}}$, refers to the apparent equilibrium constant in the photostationary state.

For the general case of "non-equilibrium" facilitation, the method of orthogonal collocation on finite elements discussed in another paper [2] can be readily applied to solve the diffusion-reaction equations in both the illuminated and the dark zones of the membrane. This method has very good convergence properties. As additional elements are placed in the region where the residuals are large, the error in the solution decreases very rapidly. In the development of the method, the independent variable and its derivatives are made continuous at the element end points. So the continuity of the concentrations and the fluxes across the interface in this problem can be readily satisfied by placing an element end point at this position. Although the procedure developed above is based on simple kinetic scheme $A+B \rightleftharpoons A B$, the computer program for the method, discussed by Jain and Schultz [2], is applicable to any general kinetic scheme. The computer calculations yield the boundary concentrations of $\mathrm{B}$. The concentrations of $\mathrm{A}$ at the boundaries are known from the boundary conditions. The flux can then be calculated using eqn. (9) below.

$N_{\mathrm{A}}=\frac{1}{L}\left[D_{\mathrm{A}}\left(C_{\mathrm{A}}^{0}-C_{\mathrm{A}}^{L}\right)+D_{\mathrm{B}}\left(C_{\mathrm{B}}^{L}-C_{\mathrm{B}}^{0}\right)\right]$

Results of the numerical simulation are discussed next.

\section{Numerical results and discussion}

The values of the physical constants for the carbon monoxide-hemoglobin system used in the numerical calculations were taken from Kutchai et al. [8]. The results of Bonaventura et al. [5] were used for the values of ap- 
parent equilibrium constant. Bonaventura et al. [5] showed that the binding affinity of $\mathrm{CO}$ to myoglobin can be reduced by a factor of over 1000 in strong light. They also suggested that similar results were expected for hemoglobincarbon monoxide system. For the present calculations, values for the ratio of the equilibrium constants in the dark and light regions, $K_{\mathrm{D}} / K_{\mathrm{L}}$, were varied from 1 to 500 for the hemoglobin-carbon monoxide system.

Figure 2 shows the flux of $\mathrm{CO}$ across a membrane of total thickness $10 \mu \mathrm{m}$ and a ratio of equilibrium constants, $K_{D} / K_{L}$, of 10 . Two independent parameters were varied in these calculations: (a) the percentage of membrane illuminated, $\left(1-L_{\mathrm{i}} / L\right) \times 100$, and (b) the concentration of permeant on the downstream side of the membrane. As might be expected, for a given fractional illumination of the membrane, the flux decreases as the downstream concentration, $C_{\mathrm{A}}^{L}$, is increased. This can readily be seen in Fig. 2. Of more interest are the maximum concentration ratios of $\mathrm{CO}$ that can be achieved across the membrane by light energy, and these values can be found where the lines intersect the $x$-axis, i.e. when the flux is zero. The maximum concentration ratio is found when the fraction illuminated is about $50 \%$.

We also see from Fig. 2 that the flux reaches maximum (for a given concentration ratio) at a value of percentage illumination between 0 and 100 .

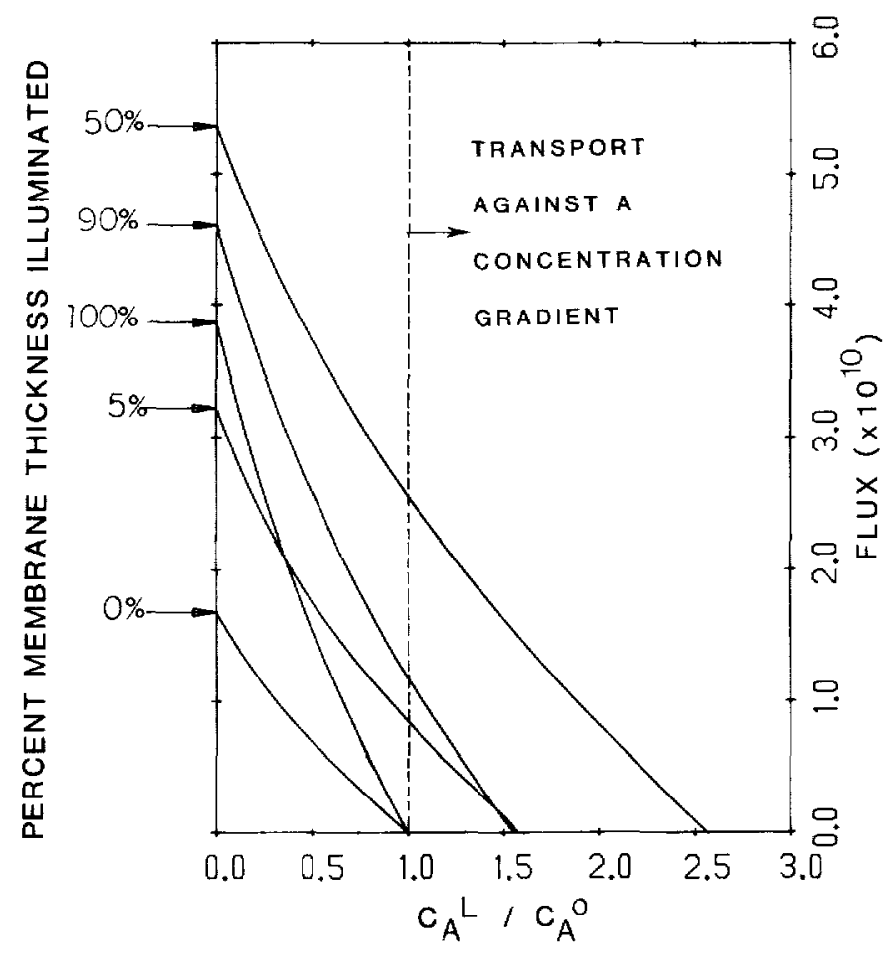

Fig. 2. Flux vs. $C_{\mathrm{A}}^{L} / C_{\mathrm{A}}^{0}$ as function of percentage illumination $\left(L=10 \mu \mathrm{m}, K_{\mathrm{R}}=10, C_{\mathrm{A}}^{0}=\right.$ $5.36 \times 10^{-9} \mathrm{~mol} / \mathrm{ml}$ ). 
The fluxes reach a maximum at 50\% illumination in Fig. 2. As will be seen later, in Fig. 6, this maximum value is obtained at different percentage illuminations for different membranes, because it depends on the membrane thickness and the ratio of the equilibrium constants. The direction of the flux in all cases is from left to right in Fig. 1, i.e. from the dark to the lighted side of the membrane. The flux results indicate that partial illumination of the membrane is more effective than either complete illumination or absence of illumination. Figure 2 also indicates that for the partially illuminated membranes, the flux values are non-zero, even when $C_{\mathrm{A}}^{L}>C_{\mathrm{A}}^{0}$, i.e. transport can be achieved even against a concentration gradient for these membranes.

As mentioned earlier, the maximum transport that can be obtained across the membrane is under the conditions of a high Damköhler number, i.e. chemical equilibrium throughout the membrane. In Fig. 3, the equilibrium and non-equilibrium flux profiles for partially illuminated (50\% illumination) and fully illuminated membranes are plotted as a function of $C_{\mathrm{A}}^{L} / C_{\mathrm{A}}^{0}$ for the conditions corresponding to Fig. 2. We see that the flux values for $50 \%$

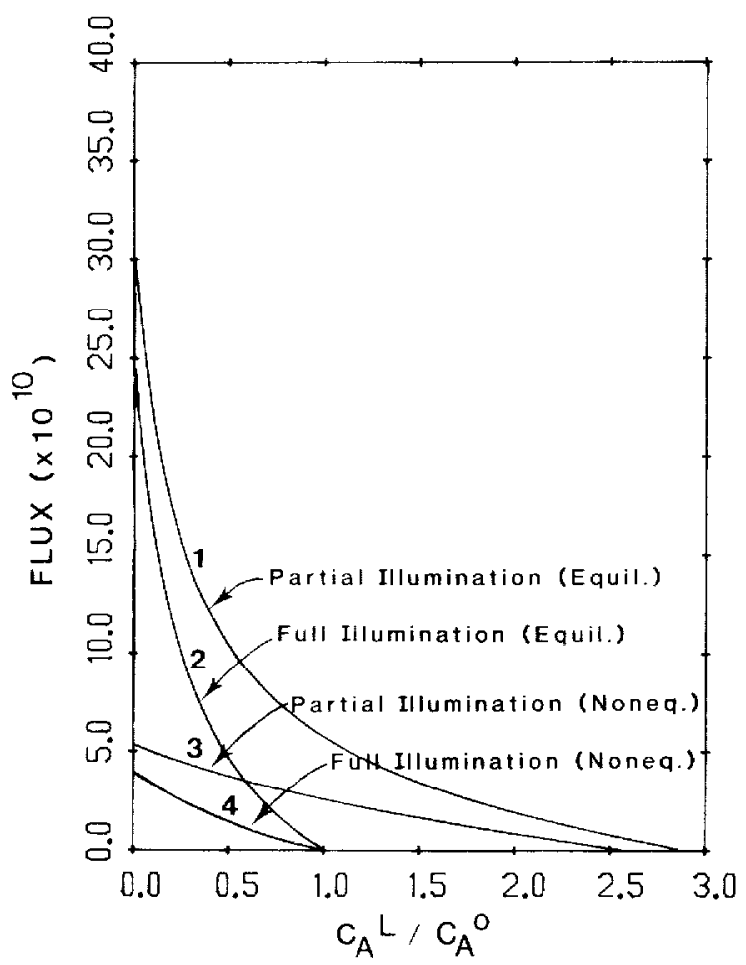

Fig. 3. Comparison of flux curves for various equilibrium and non-equilibrium conditions ( 1 , equilibrium curve for a partially illuminated membrane; 2 , equilibrium curve for a completely illuminated membrane; 3 , non equilibrium curve for a membrane with $50 \%$ illumination; 4 , non equilibrium curve for a completely illuminated membrane) with $L=$ $10 \mu \mathrm{m}, K_{\mathbf{R}}=10$ and $C_{\mathrm{A}}^{0}=5.36 \times 10^{-9} \mathrm{~mol} / \mathrm{ml}$. 
illumination are lower than the equilibrium flux values for a partially illuminated membrane, indicating thereby that we are in the non-equilibrium facilitation regime. We also see that the equilibrium flux values for partially illuminated membrane are higher than the corresponding values for an uniform ly illuminated membrane. The difference is substantial for non-zero downstream concentrations. Similar differences can be seen for the non-equilibrium case. Comparison of the equilibrium facilitation curve for a partially illuminated membrane with the non-equilibrium facilitation curve at $50 \%$ illumination indicates that as the downstream concentration, $C_{\mathrm{A}}^{L}$, is increased, the two curves become closer. This is coincident with the increased contribution of the light-induced flux to the total flux as the downstream concentration increases. Indeed, the equilibrium concentration ratio across the partially illuminated membrane at zero flux is very close to the value for the 50\% illuminated membrane. The effect of light thus seems to be to bring the system closer to equilibrium as the downstream permeant concentration increases

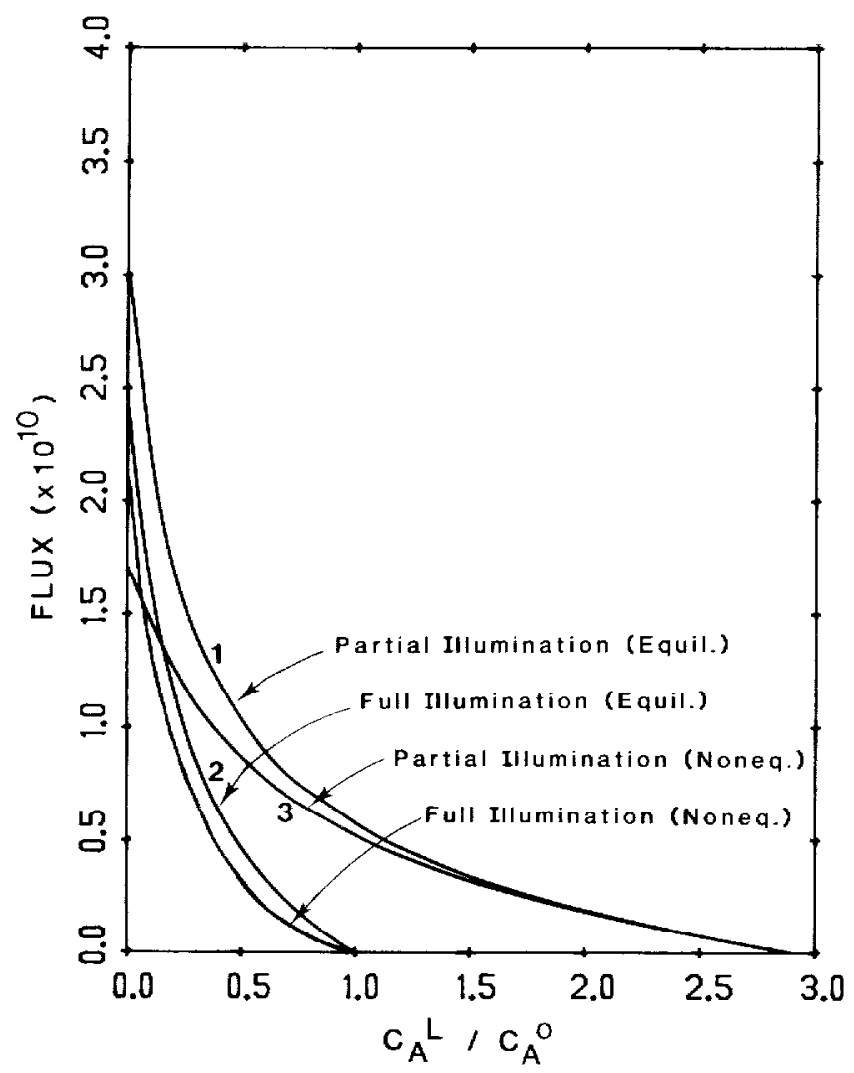

Fig. 4. Flux curves for various equilibrium and non-equilibrium conditions with $L=100$ $\mu \mathrm{m}, K_{\mathbf{R}}=10$ and $C_{\mathrm{A}}^{0}=5.36 \times 10^{-9} \mathrm{~mol} / \mathrm{ml}$. Curves 1 and 2 are same as in Fig. 3 , but the fluxes here are one-tenth as great. 
In Fig. 4, curves similar to Fig. 3 are plotted for a membrane thickness of $100 \mu \mathrm{m}$ with other conditions the same. We see that for a ratio of downstream to upstream concentration of greater than 0.5 , the equilibrium and non-equilibrium curves for a partially illuminated membrane are fairly close (curves 1 and 2). This, however, is not the case for the non-equilibrium and equilibrium curves for a completely illuminated membrane (curves 3 and 4 ). These results, along with those of Fig. 3, indicate that in carrier-mediated photodiffusion membranes (partial illumination), we not only obtain very high fluxes (compared to totally dark or illuminated membranes) but also facilitations close to the equilibrium facilitations for relatively thin membranes. The latter result applies to the case when the downstream and upstream concentrations are comparable. This result is important because the absolute flux across a membrane generally decreases with increasing the membrane thickness for both equilibrium and non-equilibrium facilitations.

The concentration profiles for the membranes corresponding to Fig. 2 are shown in Fig. 5 for various values of downstream concentrations with onehalf of the membrane illuminated. The concentration profiles seem to be wellbehaved and no sharp changes in the slope are seen, indicating that very thin boundary layers do not exist under these conditions. As discussed earlier,

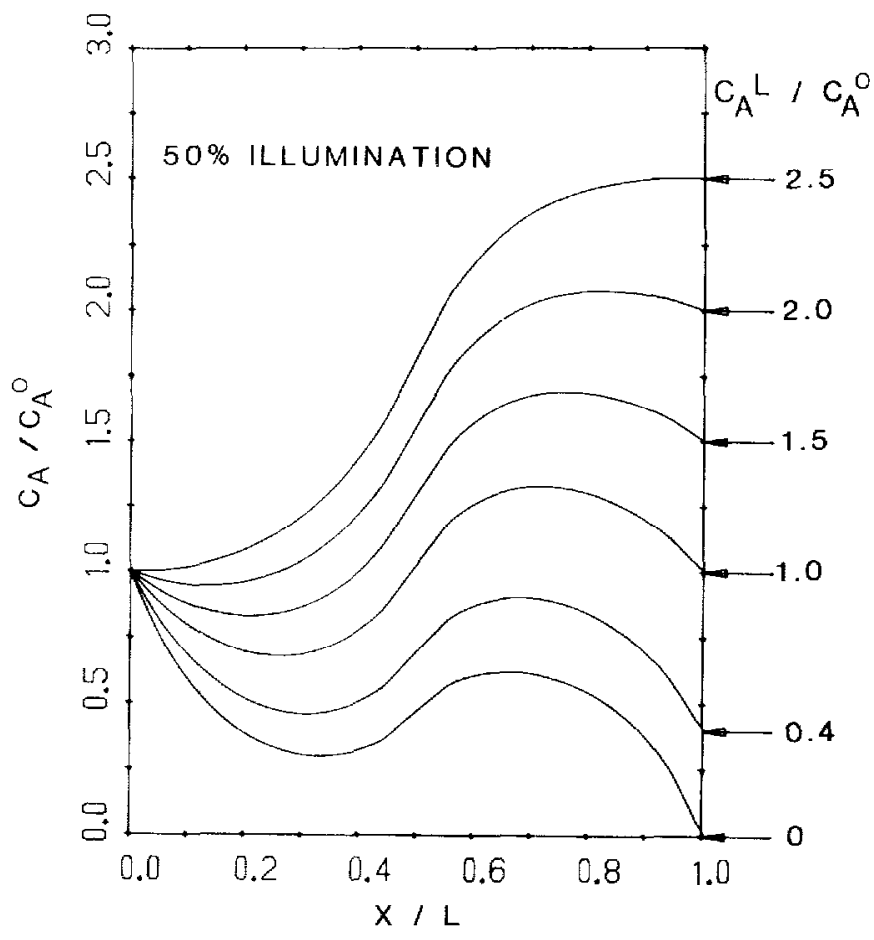

Fig. 5. Concentration profiles for the permeant as function of $C_{\mathrm{A}}^{L} / C_{\mathrm{A}}^{0}\left(L=10 \mu \mathrm{m}, K_{\mathrm{R}}=\right.$ 10 and $C_{\mathrm{A}}^{0}=5.36 \times 10^{-9} \mathrm{~mol} / \mathrm{ml}$ ). 
the system approaches equilibrium as the downstream concentration is increased. However we do not see any abrupt changes in the concentration profiles with the increase in the downstream permeant concentration. About 8 finite elements were needed to solve for fluxes for the various cases depicte in Fig. 5.

In Fig. 6, each flux curve is plotted as a function of fractional illumination for the case where the upstream and downstream concentrations are equal, i.e. $C_{\mathrm{A}}^{L}=C_{\mathrm{A}}^{0}$. Since in the absence of light the flux across the membrane will be zero in this case, this situation corresponds to one in which the transport of the permeant is solely due to the effect of light. In order to compare different modalities of illuminating the membranes, combinations of membrane thicknesses and equilibrium constant ratios were chosen for different membranes such that the total amount of light energy impinging on different membranes was the same. From various curves it is clear that the flux goes through a maximum as the percentage of membrane illuminated is changed from 0 to 100. On the basis of flux comparisons in this figure, thin membranes with high degrees of illumination (high $K_{R}$ ) seem to be more efficient at small percentage illuminations (curve 2), while membranes with inter-

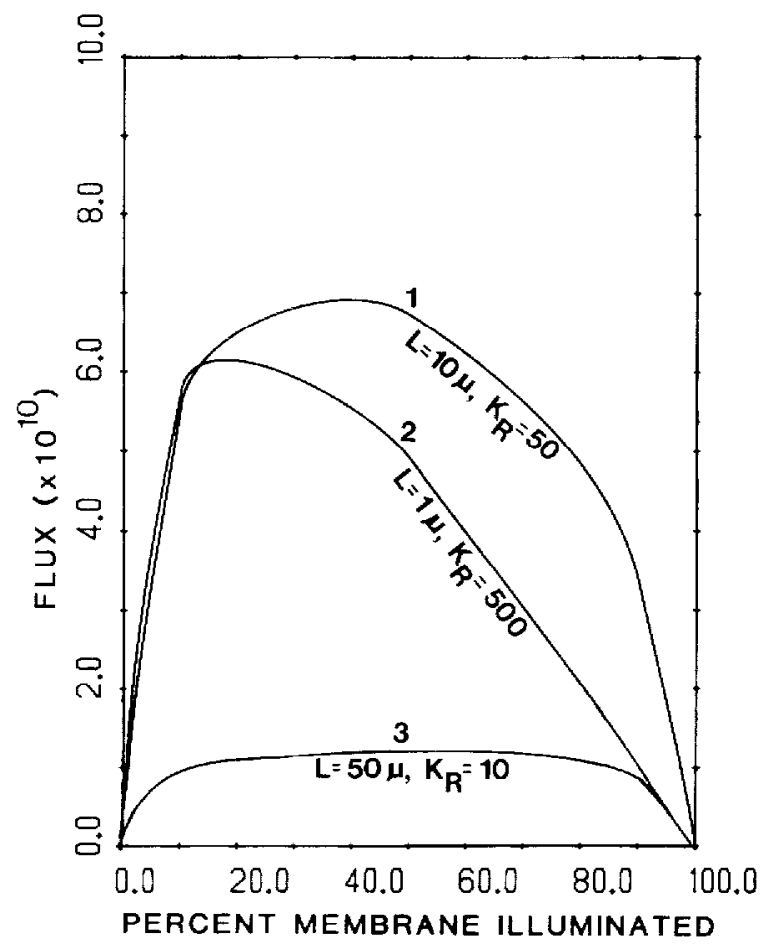

Fig. 6. Flux vs. percentage illumination as function of membrane thickness and equilibrium constant ratio $\left(1, L=10 \mu \mathrm{m}, K_{\mathrm{R}}=50 ; 2, L=1 \mu \mathrm{m}, K_{\mathrm{R}}=500 ; 3, L=50 \mu \mathrm{m}, K_{\mathrm{R}}=\right.$ 10), for $C_{\mathrm{A}}^{L}=C_{\mathrm{A}}^{0}=5.36 \times 10^{-9} \mathrm{~mol} / \mathrm{ml}$. 
mediate thickness and illumination are most efficient at larger percentage illuminations (curve 1). Thick membranes at low degrees of illumination (curve 3 ) are very inefficient for all values of percentage illumination.

Concentration profiles for the case where $C_{\mathrm{A}}^{L}=C_{\mathrm{A}}^{0}$ are shown in Fig. 7 for a membrane of thickness $10 \mu \mathrm{m}$ and $K_{\mathrm{D}} / K_{\mathrm{L}}=10$. The concentration profiles show an inversion as percentage illumination across the membrane is increased. For the most part, the concentrations in the illuminated part of the membrane are higher than the boundary concentrations. The concentration profiles are flat for 0 and $100 \%$ illumination because no transport takes place under these conditions. The computational effort for calculating these concentration profiles was about the same as for the profiles in Fig. 5.

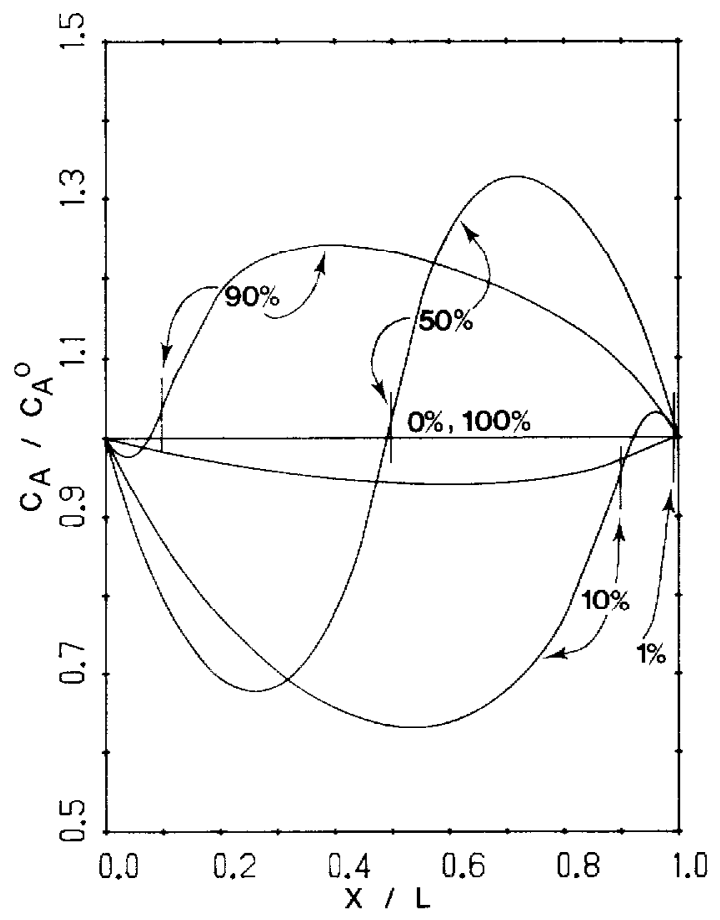

Fig. 7. Concentration profiles for the permeant as function of percentage of membrane thickness illuminated $\left(L=10 \mu \mathrm{m}, K_{\mathrm{R}}=10, C_{\mathrm{A}}^{L}=C_{\mathrm{A}}^{0}=5.36 \times 10^{-9} \mathrm{~mol} / \mathrm{ml}\right)$; vertical lines in the curves represent the boundary between dark and illuminated region.

One problem with the flux curves in Fig. 6 is that the membranes with different amounts of light impinging on them cannot be compared. To show more explicitly how an optimum utilization of light energy can be achieved, the normalized flux values for different membranes should be compared. The normalized flux is defined as the flux per unit light energy impinging on the membrane and is a measure of the efficiency of light utilization. The ratio 
of the total amount of light, $T_{\mathrm{L}}$, on two membranes with different thickness and equilibrium constant ratios, $K_{\mathrm{R}}$, is given by eqn. (10) below.

$\frac{\left(T_{\mathrm{L}}\right)_{1}}{\left(T_{\mathrm{L}}\right)_{2}}=\frac{(I L)_{1}}{(I L)_{2}}$

Now

$K_{\mathrm{R}}=\frac{K_{\mathrm{D}}}{K_{\mathrm{L}}}=\frac{k_{2}(0)+\lambda I}{k_{2}(0)}$

and since for the $\mathrm{CO}$-hemoglobin system $\lambda I \gg k_{2}(0)$, we have

$K_{\mathrm{R}}=\frac{\lambda I}{k_{2}(0)}$, or $K_{\mathrm{R}} \propto I$

so eqn. (10) reduces to

$\frac{\left(T_{\mathrm{L}}\right)_{1}}{\left(T_{\mathrm{L}}\right)_{2}}=\frac{\left(K_{\mathrm{R}} L\right)_{1}}{\left(K_{\mathrm{R}} L\right)_{2}}$

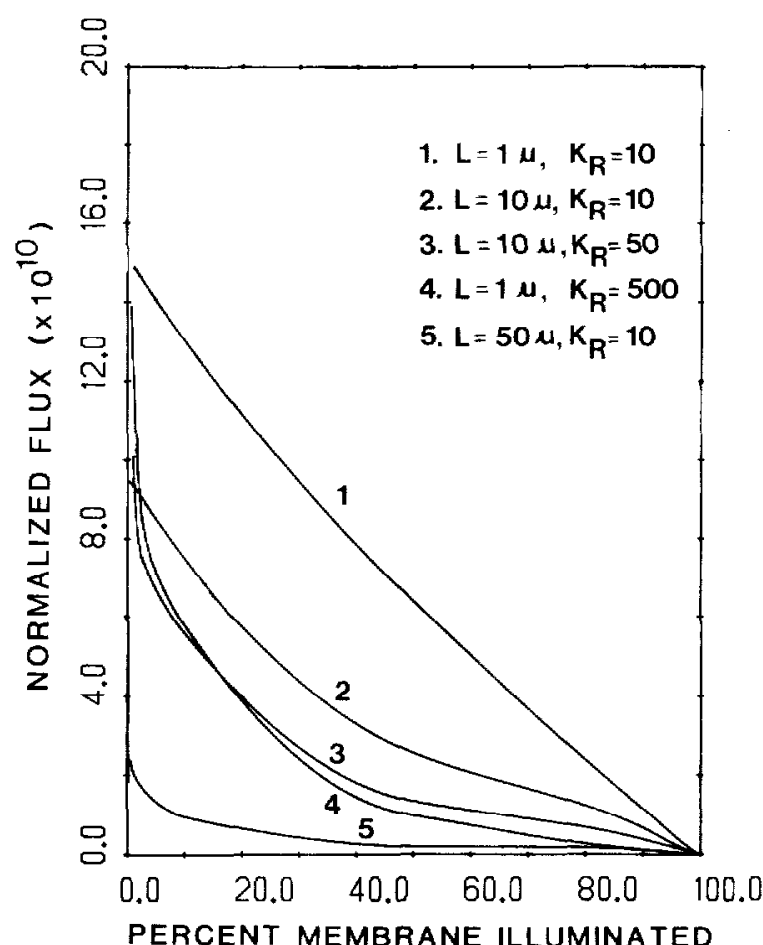

Fig. 8. Normalized flux vs. percentage of membrane illuminated for $C_{\mathrm{A}}^{L}=C_{\mathrm{A}}^{0}=5.36 \times 10$ $\mathrm{mol} / \mathrm{ml}\left(1, L=1 \mu \mathrm{m}, K_{\mathrm{R}}=10 ; 2, L=10 \mu \mathrm{m}, K_{\mathrm{R}}=10 ; 3, L=10 \mu \mathrm{m}, K_{\mathrm{R}}=50 ; 4, L=1\right.$ $\left.\mu \mathrm{m}, K_{\mathrm{R}}=500 ; 5, L=50 \mu \mathrm{m}, K_{\mathrm{R}}=10\right)$. 
Equation (12) can be used to compare the total amount of light falling on various membranes by taking one of the membranes as a reference.

Normalized flux is plotted against percentage illumination in Fig. 8. The results indicate that thin membranes at low degrees of illumination (low values of $K_{R}$ ) are most efficient (curve 1) and again thick membranes at low degrees of illumination are most inefficient (curve 5). Also, more efficient use of light energy results at low values of percentage of membrane illuminated. We also see that the optimum range of percentage illumination values is very narrow, because as percentage illumination increases the flux decreases very rapidly. These comparisons assume that the unabsorbed light can be usefully utilized, which of course may not be the case in practice. Since thin membranes would normally absorb smaller amounts of light than thick membranes, more light may be wasted in thin membranes. It may also not be possible to produce very thin membranes which are defect free; so practical con-

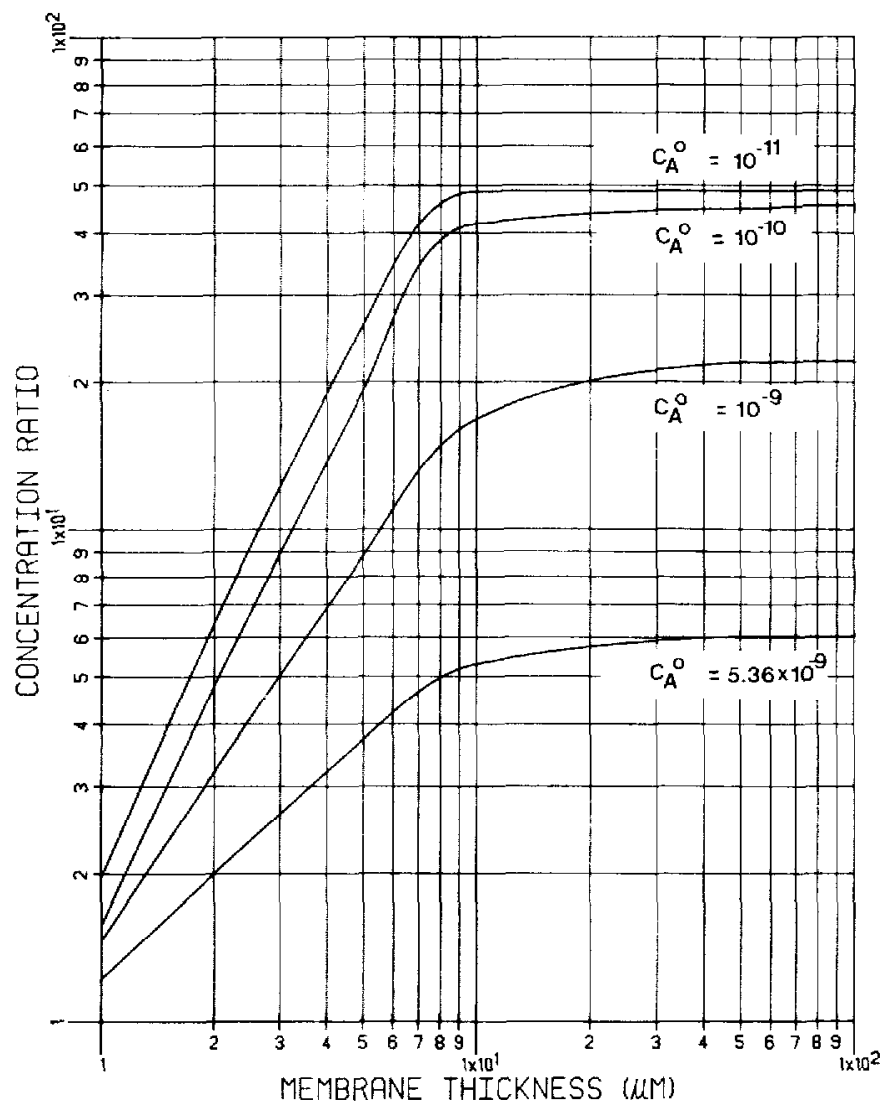

Fig. 9. Effect of upstream concentration on $\mathrm{CO}$ enrichment as function of membrane thickness $\left(K_{R}=50\right.$, percentage illumination $\left.=50\right)$. 
siderations may change the optimum conditions for the operation of these membranes.

Transport against a concentration gradient and applications in the storage of solar energy

As discussed earlier, transport in carrier-mediated photodiffusion membranes can be achieved even against a concentration gradient (Fig. 2). The limiting situation corresponds to the case in which the flux of the permeant across the membrane is zero and a concentration gradient of the permeant is established across the membrane. In Fig. 9, the concentration ratios under a condition of no flux are plotted as a function of membrane thickness with upstream concentration as a parameter. From Fig. 9, we see that concentration ratios increase very rapidly with the increases in membrane thickness and then reach a constant value. This constant value corresponds to the

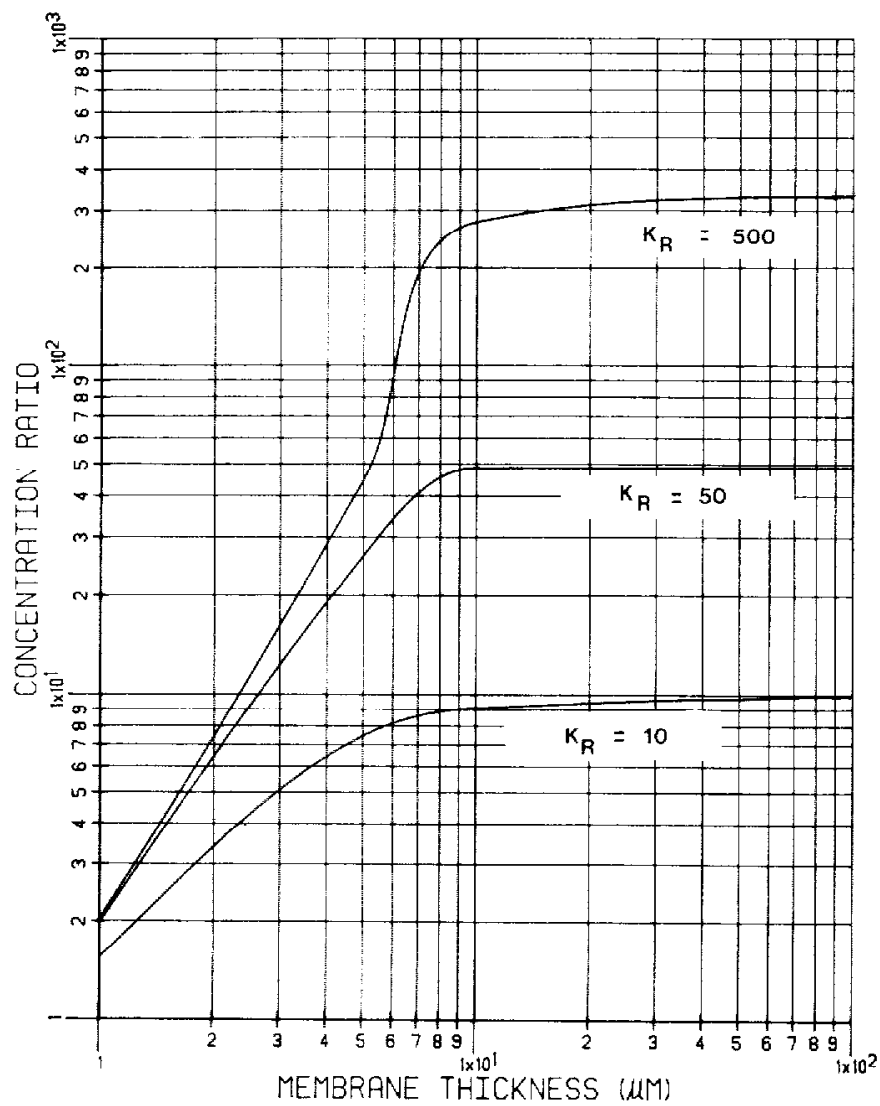

Fig. 10. Effect of light intensity on CO enrichment as function of membrane thickness (upstream concentration $=10^{-11} \mathrm{~mol} / \mathrm{ml}$, percentage illumination $=50$ ). 
"equilibrium regime" concentration ratio. We see here again, as in Figs. 3 and 4 , that the equilibrium facilitations for these membranes can be obtained at very low membrane thicknesses. We also see from Fig. 9 that as the upstream concentration decreases, the concentration ratio achievable increases. In Fig. 10, concentration ratios under no-flux condition are plotted as a function of equilibrium constant ratio for a very low upstream concentration. We see that as the equilibrium constant ratio increases, the concentration ratio that can be supported across the membrane also increases. So in order to obtain high concentration ratios across the membrane under the conditions of no flux, the membrane should have high light intensity and low upstream permeant concentration.

In a preliminary fashion we also explored how the spatial distribution of light across the membrane affects the efficiency of light utilization. As shown in Fig. 11, a step profile in light intensity within the membrane is more effective in the intermediate Damköhler regime in producing increase in permeant

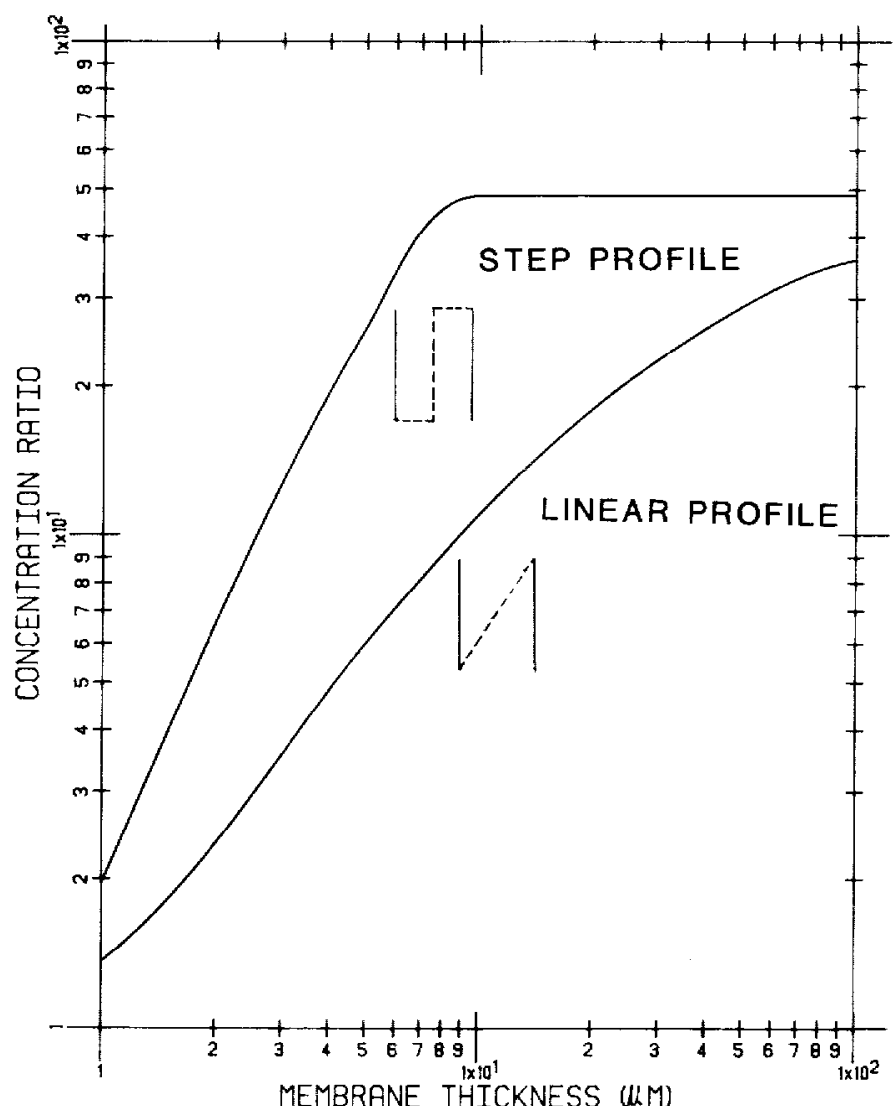

Fig. 11. Effect of light profile on enrichment ratio as function of membrane thickness (upstream concentration $=10^{-11} \mathrm{~mol} / \mathrm{ml}$, percentage illumination $=50$ ) . 
concentration across the membrane. The concentration ratio is independent of membrane thickness and illumination profile beyond a certain thickness (Damköhler number).

This high concentration gradient of the permeant can be coupled to an electrochemical reaction to produce energy using a fuel cell. This is shown schematically in Fig. 12. A prototype reaction under study for this purpose is the decomposition of nitrosyl chloride in an organic solvent. The concentration gradient of nitric oxide across the membrane can be used to run a fuel cell as discussed by McKee et al. [9] .

\section{Membrane Containing the Carrier}

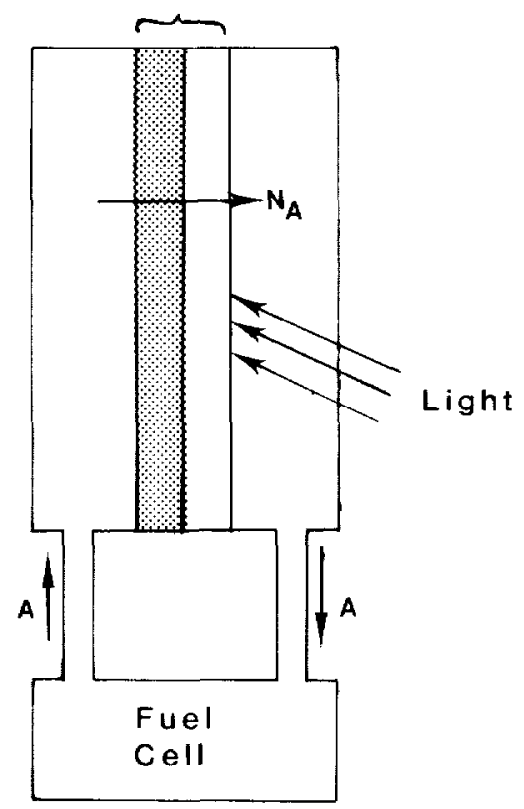

Fig. 12. Typical solar energy conversion system utilizing photodiffusion membranes.

\section{Conclusions}

The results in this paper indicate that the method of orthogonal collocation on finite elements can be successfully applied for the analysis of carriermediated photodiffusion membranes. Transport across these membranes can be achieved even in the absence of a concentration gradient. Partial illumination of the membrane increases the maximum flux values (equilibrium fluxes) that can be obtained for either totally illuminated or non-illuminated membranes. For the case of partial illumination, the effect of light is to bring the system closer to the equilibrium as the downstream permeate concentration is increased, even for very thin membranes. Based on normalized flux com- 
parisons, the indications are that the optimum utilization of light energy is obtained for thin membranes at low degrees of illumination. Carrier-mediated photodiffusion membranes can also be coupled to a fuel cell for the storage of solar energy.

\section{Acknowledgement}

This work was supported by NSF grant no. NSF/CPE 78-13316.

\section{List of symbols}

A Permeant.

AB Carrier-permeant complex.

B Carrier.

$C_{\mathrm{A}}^{0} \quad$ Permeant concentration at $x=0(\mathrm{~mol} / \mathrm{ml})$.

$C_{\mathrm{A}}^{L} \quad$ Permeant concentration at $x=L(\mathrm{~mol} / \mathrm{ml})$.

$C_{i}^{\mathrm{A}} \quad$ Concentration of species $i(i=\mathrm{A}, \mathrm{B}, \mathrm{AB})$ in the illuminated side of the membrane $(\mathrm{mol} / \mathrm{ml})$.

$\bar{C}_{i} \quad$ Concentration of species $i(i=\mathrm{A}, \mathrm{B}, \mathrm{AB})$ in the dark side of the membrane $(\mathrm{mol} / \mathrm{ml})$.

$C_{\mathrm{T}} \quad$ Total carrier concentration in all its forms $(\mathrm{mol} / \mathrm{ml})$.

$D_{i} \quad$ Diffusivity of species $i(i=\mathrm{A}, \mathrm{B}, \mathrm{AB})\left(\mathrm{cm}^{2} / \mathrm{sec}\right)$.

I Light intensity.

$K \quad$ Equilibrium constant of the reaction $(\mathrm{ml} / \mathrm{mol})$.

$k_{1} \quad$ Forward rate constant of the reaction $(\mathrm{ml} / \mathrm{mol}-\mathrm{sec})$.

$k_{2} \quad$ Reverse rate constant of the reaction $\left(\mathrm{sec}^{-1}\right)$.

$k_{2}(I) \quad$ Reverse rate constant of the reaction in light of intensity $I\left(\mathrm{sec}^{-1}\right)$.

$k_{2}(0) \quad$ Reverse rate constant of the reaction in the dark $\left(\mathrm{sec}^{-1}\right)$.

$K_{\mathrm{D}} \quad$ Equilibrium constant of the reaction in the dark $(\mathrm{ml} / \mathrm{mol})$.

$K_{\mathrm{L}} \quad$ Apparent equilibrium constant of the reaction in light (the photostationary state) $(\mathrm{ml} / \mathrm{mol})$.

$K_{\mathrm{R}} \quad$ Equilibrium constant ratio $\left(=K_{\mathrm{D}} / K_{\mathrm{L}}\right)$.

$L \quad$ Membrane thickness $(\mu \mathrm{m})$.

$L_{\mathbf{i}} \quad$ Thickness of the dark part of the membrane $(\mu \mathrm{m})$.

$N_{\mathrm{A}} \quad$ Flux of the permeant across the membrane $\left(\mathrm{mol} / \mathrm{cm}^{2}\right.$-sec $)$.

$N_{\mathrm{D}} \quad$ Damköhler number $\left(=k_{2} L^{2} / D_{\mathrm{A}}\right)$.

$r_{i} \quad$ Reaction rate for component $i$ in the dark part of the membrane ( $i=$ $\mathrm{A}, \mathrm{B}, \mathrm{AB})(\mathrm{mol} / \mathrm{ml}-\mathrm{sec})$.

$\bar{r}_{i} \quad$ Reaction rate for component $i$ in the illuminated part of the membrane $(i=\mathrm{A}, \mathrm{B}, \mathrm{AB})(\mathrm{mol} / \mathrm{ml}-\mathrm{sec})$.

$T_{\mathbf{L}} \quad$ Total amount of light energy falling on the membrane.

$x \quad$ Distance coordinate along the membrane thickness.

$\lambda$ Proportionality constant in the expression for $k_{2}(I)\left(=k_{2}(0)+\lambda I\right)$. 


\section{References}

1 J.S. Schultz, Carrier-mediated photodiffusion membranes, Science, 197 (1977) 1177.

2 R. Jain and J.S. Schultz, A numerical analysis of carrier-mediated transport, J. Membrane Sci., 11 (1982) 79.

3 T.L. Donaldson and J.A, Quinn, Carbon dioxide transport through enzymatically active synthetic membranes, Chem. Eng. Sci., 30 (1975) 103.

4 J.S. Schultz, Facilitation of $\mathrm{CO}_{2}$ through layers with a spatial distribution of carbonic anhydrase, in: C. Bauer, G. Gros and H. Bartels (Eds.), Biophysics and Physiology of Carbon Dioxide, Springer-Verlag, Berlin, 1980.

5 C. Bonaventura, J. Bonaventura, E. Antonini, M. Brunori and J. Wyman, Carbon monoxide binding by simple hemeproteins under photodissociating conditions, Biochem., 12 (1973) 3424.

6 K.A. Smith, J.H. Meldon and C.K. Colton, An analysis of carrier-facilitated transport, AIChE J., 19 (1973) 102.

7 J.S. Schultz, J.D. Goddard and S.R. Suchdeo, Facilitated transport via carrier-mediated diffusion, Part I, Mechanistic aspects, experimental systems and characteristic regimes, AIChE J., 20 (1974) 417.

8 H. Kutchai, J.A. Jacquez and F.J. Mather, Nonequilibrium facilitated oxygen transport in hemoglobin solutions, Biophys. J., 10 (1970) 38.

9 W.E. McKee, E. Findl, J.D. Margerum and W.B. Lee, A nitrosyl chloride solar regenerative fuel cell system, Wright Air Development Division (WADD) Tech. Rep, 60-821, part I and II, 1961. 